\title{
Quasipinning and entanglement in the lithium isoelectronic series
}

\author{
Carlos L. Benavides-Riveros, ${ }^{1,2,3,{ }^{*}}$ José M. Gracia-Bondía, ${ }^{1,2}$ and Michael Springborg ${ }^{3}$ \\ ${ }^{1}$ Departamento de Física Teórica, Universidad de Zaragoza, 50009 Zaragoza, Spain \\ ${ }^{2}$ Instituto de Biocomputación y Física de Sistemas Complejos (BIFI), Universidad de Zaragoza, 50018 Zaragoza, Spain \\ ${ }^{3}$ Physikalische und Theoretische Chemie, Universität des Saarlandes, 66123 Saarbrücken, Germany
}

(Received 27 June 2013; published 19 August 2013)

\begin{abstract}
The Pauli exclusion principle gives an upper bound of 1 on natural occupation numbers. Recently there has been an intriguing amount of theoretical evidence that there is a plethora of additional generalized Pauli restrictions or (in)equalities, of a kinematic nature, satisfied by these numbers [M. Altunbulak and A. Klyachko, Commun. Math. Phys. 282, 287 (2008)]. Here a numerical analysis of the nature of such constraints is effected in real atoms. The inequalities are nearly saturated, or quasipinned. For rank 6 and rank 7 approximations for lithium, the deviation from saturation is smaller than the lowest occupancy number. For a rank 8 approximation we find well-defined families of saturation conditions.
\end{abstract}

DOI: 10.1103/PhysRevA.88.022508

PACS number(s): 31.15.V-, 03.67.-a

\section{INTRODUCTION}

The natural occupation numbers, arranged in the customary decreasing order $\lambda_{1} \geqslant \lambda_{2} \geqslant \cdots$, fulfill $0 \leqslant \lambda_{i} \leqslant 1$ for all $i-$ thus allowing no more than one electron in each quantum state. Forty years ago Borland and Dennis [1] observed for the rank 6 approximation of a three-electron system, whose state space is here denoted $\wedge^{3} \mathcal{H}_{6}$, that the six occupation numbers satisfy the additional constraints $\lambda_{r}+\lambda_{7-r}=1$, where $r \in\{1,2,3\}$, allowing exactly one electron in the natural orbitals $r$ and $7-r$. Moreover,

$$
\lambda_{4} \leqslant \lambda_{5}+\lambda_{6}
$$

The proofs are given in [2]. Thanks to outstanding work by Klyachko and others in the last few years, actually solving the pure-state $N$-representability problem for the one-body reduced density matrix [3], the pattern of the occupation numbers has received renewed attention. Large sets of inequalities for the eigenvalues of this matrix, widely generalizing (1), have been established. We note that, while the pure $N$-representability problem for the two-body reduced density matrix remains unsolved, the ensemble $N$-representability problem for this matrix is now solved [4].

A recent article [5] proposes to carry out an analytic study of the Klyachko conditions by means of a toy model: a onedimensional system of three spinless fermions confined to a harmonic well, interacting with each other through Hooke-type forces. A series formula for the occupation numbers in terms of the coupling was found.

The tantalizing suggestion in [5] is that the inequalities are nearly saturated in the ground state [i.e., in equations like Eq. (1) the equality almost holds]: this is the "quasipinning" phenomenon, which points to a deep hold on the kinematics of the system. Schilling et al. [5] state, “...It is likely extremely challenging to use numerical methods to distinguish between genuinely pinned and mere quasi-pinned states."

In the work we report here, we have taken up this challenge by studying the ground state of lithium-like ions, starting

\footnotetext{
*carlosbe@unizar.es
}

from scratch with an elementary configuration interaction $(\mathrm{CI})$ method, up to a rank 8 approximation (here, the rank equals the number of basis functions in setting up the CI expansion). This procedure serves a twofold purpose. First, we study whether the conclusions of Schilling et al. [5] are valid for realistic systems too. There now exists a profound measure of quantum entanglement for three-fermion systems in rank 6 [6]. The second goal of the present work is therefore to adapt this measure to our physical spin-partitioned systems, contrasting the results with the information on entanglement traditionally provided by the $\lambda_{i}$.

In the present paper we present our analysis and results as follows. Section II gives a simple introduction to the problem at hand. We discuss in some detail the one- and two-body matrices in the relatively trivial approximation of rank 5 to the lithium-like ground states $\wedge^{3} \mathcal{H}_{5}$.

In Section III we broach the subject of entanglement for our systems. This allows us to discuss subsequently the information-theoretic meaning of pinning and quasipinning.

Section IV deals with the first nontrivial approximation to the three-electron system (of rank 6). We use two different basis sets, and the comparison of the results turns out to be very instructive.

Section V analyzes the more complicated cases of rank 7 and 8 approximations. Finally, Sec. VI summarizes our conclusion.

We emphasize that the calculated energies are used merely to provide information on the quality of our approximations and, accordingly, on how accurate our calculated occupation numbers are, including our conclusions with regard to the quasipinning hypothesis. Our goal is to grapple with its impact on chemistry and to investigate the negative correlation between it and entanglement. Thus we refrain completely from gaining the extra accuracy of machine calculations at the price of losing insight.

In the two appendixes we give some additional mathematical information. Appendix A discusses the ideas behind the Klyachko constraints in ordinary quantum chemical language. Appendix B gives the proof of an estimate that we present in Sec. V.

Finally, throughout this work we use Hartree atomic units. 


\section{THE SIMPLEST CASE: A RANK 5 CONFIGURATION FOR LITHIUM-LIKES}

Consider a system of $N$ electrons and $M$ spin orbitals $\left\{\varphi_{i}(\boldsymbol{x})\right\}_{i=1}^{M}$, each being a product of a spatial orbital and a spinor. We employ the standard quantum-chemical notation $\boldsymbol{x}:=(\boldsymbol{r}, \varsigma)$ and use the notational convention: $\varphi_{i}(\boldsymbol{x}):=$ $\phi_{i}(\boldsymbol{r}) \varsigma$, with $\varsigma \in\{\uparrow, \downarrow\}$. The number of configurations $N_{c}$ that can be constructed from $M$ spin orbitals for $N$ electrons and $M-N$ holes is

$$
N_{c}=\left(\begin{array}{c}
M \\
N
\end{array}\right)
$$

which grows as a factorial with $M$. Here, we assume that we have identified a set of basis functions, largely under the guidance of the physical or chemical intuition [7], which provides an accurate description of the system of our interest. For the $N$-electron wave function, we use wave functions made of normalized Slater determinants,

$$
|\Psi\rangle=\sum_{J} C_{J}\left[\varphi_{J(1)} \cdots \varphi_{J(N)}\right] .
$$

With the exterior algebra notation, this becomes

$$
\left[\varphi_{1} \varphi_{2} \cdots \varphi_{N}\right]=: \frac{1}{\sqrt{N !}}\left|\varphi_{1}\right\rangle \wedge\left|\varphi_{2}\right\rangle \wedge \cdots \wedge\left|\varphi_{N}\right\rangle .
$$

In general, we assume that the $\varphi_{i}$ have been orthonormalized, although we occasionally relate them to nonorthogonal orbitals by

$$
\varphi_{i}(\boldsymbol{x})=\sum_{j=1}^{L} R_{i j} \psi_{j}(\boldsymbol{r}, \varsigma) .
$$

We define the following energy integrals:

$$
\begin{aligned}
\kappa_{m n} & :=\int \frac{\varphi_{m}(\boldsymbol{x}) \varphi_{n}(\boldsymbol{x})}{|\boldsymbol{r}|} d \boldsymbol{x}, \\
\pi_{m n} & :=-\frac{1}{2} \int \varphi_{m}(\boldsymbol{x}) \nabla_{\boldsymbol{r}}^{2} \varphi_{n}(\boldsymbol{x}) d \boldsymbol{x}, \\
\iota_{m n o p} & :=\int \frac{\varphi_{m}\left(\boldsymbol{x}_{1}\right) \varphi_{n}\left(\boldsymbol{x}_{1}\right) \varphi_{o}\left(\boldsymbol{x}_{2}\right) \varphi_{p}\left(\boldsymbol{x}_{2}\right)}{\left|\boldsymbol{r}_{1}-\boldsymbol{r}_{2}\right|} d \boldsymbol{x}_{1} d \boldsymbol{x}_{2}, \\
K_{m n} & :=\int \frac{\psi_{m}(\boldsymbol{x}) \psi_{n}(\boldsymbol{x})}{|\boldsymbol{r}|} d \boldsymbol{x}, \\
P_{m n} & :=-\frac{1}{2} \int \psi_{m}(\boldsymbol{x}) \nabla_{\boldsymbol{r}}^{2} \psi_{n}(\boldsymbol{x}) d \boldsymbol{x}, \\
\Upsilon_{m n o p} & :=\int \frac{\psi_{m}\left(\boldsymbol{x}_{1}\right) \psi_{n}\left(\boldsymbol{x}_{1}\right) \psi_{o}\left(\boldsymbol{x}_{2}\right) \psi_{p}\left(\boldsymbol{x}_{2}\right)}{\left|\boldsymbol{r}_{1}-\boldsymbol{r}_{2}\right|} d \boldsymbol{x}_{1} d \boldsymbol{x}_{2} .
\end{aligned}
$$

From one set of integrals one can construct other sets by means of the relations $\kappa=(R \otimes R) K, \pi=(R \otimes R) P$, and $\iota=$ $(R \otimes R \otimes R \otimes R) \Upsilon$, where $R$ is the transformation matrix in (2).

\section{A. A simple starting configuration}

Given its low ionization potential ( $\simeq 0.198$ a.u.), it is natural to explore radial configurations of the open-shell lithium atom with a single-determinant composition of (a) two restricted helium-like spin orbitals - in turn motivated by the classical analysis by Shull and Löwdin [8] of the natural orbitals for spin
TABLE I. The exact, HF, and variational energy of Li in a singledeterminant configuration. Note the more substantial screening of the outer electron by the inner ones when including $\phi_{3}^{p}$ in the basis.

\begin{tabular}{lccc}
\hline \hline Configuration & Energy (a.u.) & $\alpha$ & $\gamma$ \\
\hline "Exact" & -7.478060 & - & - \\
$\mathrm{HF}$ & -7.432727 & - & - \\
{$\left[\psi_{1} \downarrow \psi_{1} \uparrow \psi_{3}^{s} \downarrow\right]$} & -7.393597 & 2.679747 & 1.868327 \\
{$\left[\psi_{1} \downarrow \psi_{1} \uparrow \psi_{3}^{d} \downarrow\right]$} & -7.416163 & 2.691551 & 1.892738 \\
{$\left[\psi_{1} \downarrow \psi_{1} \uparrow \psi_{3}^{p} \downarrow\right]$} & -7.417919 & 2.686435 & 1.274552 \\
\hline \hline
\end{tabular}

singlet states of $\mathrm{He}$ - and (b) one hydrogen-like, in a suitably general sense. Specifically, in such a single configuration we use the Kellner ansatz for the helium-like functions,

$$
\psi_{1}(\alpha, \boldsymbol{r})=\sqrt{\frac{\alpha^{3}}{\pi}} e^{-\alpha r} .
$$

For the spinor of the hydrogen-like function we have arbitrarily chosen $\downarrow$. For the spatial orbital, typical textbook calculations can be used for the $s$ orbital in the $L$ shell:

$\psi_{3}^{s}(\gamma, \boldsymbol{r})=\frac{1}{4} \sqrt{\frac{\gamma^{3}}{2 \pi}} L_{1}^{1}(\gamma r) e^{-\gamma r / 2}=\frac{1}{4} \sqrt{\frac{\gamma^{3}}{2 \pi}}(2-\gamma r) e^{-\gamma r / 2}$.

Moreover, we consider also the following functions:

$$
\begin{aligned}
& \psi_{3}^{p}(\gamma, \boldsymbol{r})=\frac{1}{4} \sqrt{\frac{\gamma^{5}}{6 \pi}} r e^{-\gamma r / 2} \text { and } \\
& \psi_{3}^{d}(\gamma, \boldsymbol{r})=\frac{1}{8} \sqrt{\frac{\gamma^{7}}{45 \pi}} r^{2} e^{-\gamma r / 2} .
\end{aligned}
$$

With these functions we obtain better results than with $\psi_{3}^{s}$; see Table I. The better approximation among the three, which includes $\psi_{3}^{p}$, leads to a total energy that equals $99.19 \%$ of the "exact" value. Compared to the Hartree-Fock (HF) energy given by the "best" Slater determinant, the error is less than $0.2 \%$-much more satisfactory than the Kellner approximation for helium.

For higher $Z$ in the lithium series, the accuracy naturally improves, although we do not discuss this issue further here. Note instead that the $R$ matrix mentioned above is just a GramSchmidt orthonormalization matrix, i.e.,

$$
\begin{aligned}
\left(\begin{array}{c}
\phi_{1} \uparrow \\
\phi_{1} \downarrow \\
\phi_{3} \downarrow
\end{array}\right) & =R\left(\begin{array}{c}
\psi_{1} \uparrow \\
\psi_{1} \downarrow \\
\psi_{3} \downarrow
\end{array}\right) \text {, where } \\
R & =\left(\begin{array}{ccc}
1 & 0 & 0 \\
0 & 1 & 0 \\
0 & -\frac{\left\langle\psi_{1} \mid \psi_{3}\right\rangle}{\sqrt{1-\left|\left\langle\psi_{1} \mid \psi_{3}\right\rangle\right|^{2}}} & \frac{1}{\sqrt{1-\left|\left\langle\psi_{1} \mid \psi_{3}\right\rangle\right|^{2}}}
\end{array}\right) .
\end{aligned}
$$

In order to simplify the presentation, we do not give below the explicit forms of such matrices.

\section{B. The rank 5 computation}

We obtain the rank 5 approximation by using two heliumlike one-particle wave functions and one hydrogen-like. Still being guided by [8], for the former we add the following 
function of the set (orthonormal on the ordinary space):

$$
\delta_{n}(r):=D_{n} \sqrt{\frac{\alpha^{3}}{\pi}} L_{n-1}^{2}(2 \alpha r) e^{-\alpha r}, \quad n=1,2, \ldots,
$$

where $D_{n}^{-2}=\left(\begin{array}{c}n-1 \\ 2\end{array}\right)$, and the associated Laguerre polynomials $L_{n}^{\zeta}$ are as defined in [9]. We thus have

$$
\delta_{2}(\alpha, r):=\sqrt{\frac{\alpha^{3}}{3 \pi}} L_{1}^{2}(2 \alpha r) e^{-\alpha r} .
$$

We adopt the following notation for an orthonormalized basis set of the restricted spin-orbital type:

$$
\begin{aligned}
& |1\rangle:=\varphi_{3}^{p} \downarrow, \quad|2\rangle:=\delta_{1} \downarrow, \quad|3\rangle:=\delta_{2} \downarrow, \\
& |4\rangle:=\delta_{1} \uparrow, \quad|5\rangle:=\delta_{2} \uparrow,
\end{aligned}
$$$$
\text { where }\left(\begin{array}{c}
|1\rangle \\
|2\rangle \\
|3\rangle \\
|4\rangle \\
|5\rangle
\end{array}\right)=R\left(\begin{array}{c}
\psi_{3}^{p} \downarrow \\
\delta_{1} \downarrow \\
\delta_{2} \downarrow \\
\delta_{1} \uparrow \\
\delta_{2} \uparrow
\end{array}\right) \text {. }
$$

With rank 5 , one has, in principle, $10=\left(\begin{array}{l}5 \\ 3\end{array}\right)$ Slater determinants. However, since the adopted Hamiltonian is independent of the spin coordinates, only pure spin states are physically meaningful. Obviously, there are only six determinants which are eigenvectors of the operator $S_{z}$, namely,

$$
\text { [124], [134], [125], [135], [234], [235]. }
$$

The total spin operator $S^{2}$ can be written as $S_{-} S_{+}+S_{z}+$ $S_{z}^{2}$. It is clear that the states in (4) are eigenstates of the operator $S_{z}$ (and, consequently, of $S_{z}^{2}$ ). However, it is less clear whether they are eigenstates of $S_{-} S_{+}$too. It is easy to show that the wave function

$$
\begin{aligned}
|\Psi\rangle= & A[124]+B[134]+C[125]+D[135] \\
& +E[234]+F[235]
\end{aligned}
$$

satisfies

$$
S_{-} S_{+}|\Psi\rangle-|\Psi\rangle \propto(B-C)\left([134]+\left[1^{\prime} 23\right]+[125]\right),
$$

where $\left|1^{\prime}\right\rangle$ is a spin-up counterpart of $|1\rangle$. Therefore $S_{-} S_{+}|\Psi\rangle=|\Psi\rangle$ and $S^{2}|\Psi\rangle=\frac{3}{4}|\Psi\rangle$ if and only if $B=C$.

Throughout the remainder of this paper, we have used a similar approach to identify those spin-adapted combinations of Slater determinants that are eigenfunctions to $S^{2}$ and, accordingly, are not "spin-contaminated" states.

Finally, the normalized wave function is written as

$$
\begin{aligned}
& A[124]+B[125]+B[134]+D[135]+E[234]+F[235], \\
& \text { with }|A|^{2}+2|B|^{2}+|D|^{2}+|E|^{2}+|F|^{2}=1 .
\end{aligned}
$$

With rows and columns indexed by $\{1, \ldots, 5\}$, the corresponding one-body density matrix is expressed by the matrix

$$
\left(\begin{array}{ccccc}
|A|^{2}+2|B|^{2}+|D|^{2} & B E^{*}+D F^{*} & -A E^{*}-B F^{*} & 0 & 0 \\
B^{*} E+D^{*} F & |A|^{2}+|B|^{2}+|E|^{2}+|F|^{2} & A B^{*}+B D^{*} & 0 & 0 \\
-A^{*} E-B^{*} F & A^{*} B+B^{*} D & |B|^{2}+|D|^{2}+|E|^{2}+|F|^{2} & 0 & 0 \\
0 & 0 & 0 & |A|^{2}+|B|^{2}+|E|^{2} & A B^{*}+B D^{*}+E F^{*} \\
0 & 0 & 0 & A^{*} B+B^{*} D+E^{*} F & |B|^{2}+|D|^{2}+|F|^{2}
\end{array}\right) .
$$

In our case,

$$
\rho_{1}\left(x_{1}, x_{1}^{\prime}\right)=3 \int \Psi\left(x_{1}, x_{2}, x_{3}\right) \Psi^{*}\left(x_{1}^{\prime}, x_{2}, x_{3}\right) d x_{2} d x_{3} .
$$

We can now conclude that only combinations of the form $|[a b c]\rangle\langle[d b c]|$ will contribute (where the order of $a, b$, and $c$, as well as of $d, b$, and $c$, can be changed when simultaneously taking the appropriate signs into account). For instance, $|[124]\rangle\langle[125]|$ contributes with $A B^{*}$ to the 45 matrix entry, $|[134]\rangle\langle[234]|$ contributes with $-B E^{*}$ to the 12 entry, and so on. Note that the trace of this matrix is equal to 3 , as it should be. ${ }^{1}$

We thus have $\lambda_{1}+\lambda_{2}+\lambda_{3^{\prime}}=2$ and $\lambda_{4^{\prime}}+\lambda_{5}=1$ for the natural occupation numbers; the primes in the notation are

\footnotetext{
${ }^{1}$ This is the result of the global multiplication by a factor equal to the number of electrons, as well as the division by 3 !, coming from the appropriate constant of the determinants, and the fact that each multiplication of two Slater terms contributes twice.
}

due to their not yet being in decreasing order. By definition, in the basis of natural orbitals $\left\{\left|\alpha_{i}\right\rangle\right\}$, the one-body density matrix is diagonal: $\rho_{1}=\sum_{i=1}^{5} \lambda_{i}\left|\alpha_{i}\right\rangle\left\langle\alpha_{i}\right|$, already assuming that the occupation numbers are arranged in decreasing order by interchanging $\lambda_{3^{\prime}}$ with $\lambda_{4^{\prime}}$. Therefore, it is evident that a strong selection rule applies: we can rewrite the wave function for a three-electron system in rank 5 in terms of only two configurations:

$$
\begin{aligned}
|\Psi\rangle_{3,5} & =a\left[\alpha_{1} \alpha_{2} \alpha_{3}\right]+d\left[\alpha_{1} \alpha_{4} \alpha_{5}\right], \quad|a|^{2}+|d|^{2}=1, \\
\lambda_{2} & =\lambda_{3}=|a|^{2} \geqslant|d|^{2}=\lambda_{4}=\lambda_{5} .
\end{aligned}
$$

Through this example we have given a simple proof of a theorem stated by Coleman [10]. A more sophisticated proof is found in Corollary 2 of [11].

\section{Spectral analysis of the $\boldsymbol{n}$-body and $\boldsymbol{n}$-hole density matrices on $\wedge^{3} \mathcal{H}_{5}$}

According to the Schmidt-Carlson-Keller duality [10], when applied to a three-electron system, the nonzero 
eigenvalues as well as their multiplicities are the same for the one- and the two-body matrices, i.e.,

$$
\begin{aligned}
\rho_{2} & =\sum_{i=1}^{5} \lambda_{i}\left|\omega_{i}\right\rangle\left\langle\omega_{i}\right|, \text { where } \\
c_{j}\left|\omega_{j}\right\rangle & :=3 \int \Psi\left(\boldsymbol{x}_{1}, \boldsymbol{x}_{2}, \boldsymbol{x}_{3}\right) \alpha_{j}^{*}\left(\boldsymbol{x}_{3}\right) d \boldsymbol{x}_{3} \text { with }\left|c_{j}\right|^{2}=\lambda_{j} .
\end{aligned}
$$

Thus, the eigenvectors of the two-body matrix associated with the wave function, (6), are given by

$$
\begin{aligned}
& \left|\omega_{1}\right\rangle=a\left[\alpha_{2} \alpha_{3}\right]+d\left[\alpha_{4} \alpha_{5}\right], \quad\left|\omega_{2}\right\rangle=\left[\alpha_{1} \alpha_{3}\right], \\
& \left|\omega_{3}\right\rangle=\left[\alpha_{1} \alpha_{2}\right], \quad\left|\omega_{4}\right\rangle=\left[\alpha_{1} \alpha_{5}\right], \quad\left|\omega_{5}\right\rangle=\left[\alpha_{1} \alpha_{4}\right] .
\end{aligned}
$$

For a system of $N$ particles and $M-N$ holes, the $n$ hole matrix is Hermitian and antisymmetric in each set of subindices, similar to what is the case for the $n$-particle matrix. Additionally, it satisfies the normalization conditions and sum rules:

$$
\operatorname{Tr} \eta_{n}=\left(\begin{array}{c}
M-N \\
n
\end{array}\right), \quad \int \eta_{n} d \boldsymbol{x}_{n}=\frac{M-N-n}{n} \eta_{n-1} .
$$

In the natural orbital basis, the one-hole matrix becomes

$$
\eta_{1}=\sum_{i=1}^{M}\left(1-\lambda_{i}\right)\left|\alpha_{i}\right\rangle\left\langle\alpha_{i}\right|, \quad \text { with } \quad \operatorname{Tr} \eta_{1}=M-N,
$$

i.e., $M-N=5-3=2$ in our case; while the two-hole matrix is the $Q$ matrix of lore, which, for the lithium in the rank 5 approximation, is

$$
\eta_{2}=\sum_{i=1}^{5} \mu_{i}\left|h_{i}\right\rangle\left\langle h_{i}|=| h_{1}\right\rangle\left\langle h_{1}\right|,
$$

where $\mu_{i}=0$ if $\left|\omega_{i}\right\rangle$ is a single determinant and otherwise $\mu_{i}=\lambda_{i}$. Here, $\left|h_{1}\right\rangle:=d\left[\alpha_{2} \alpha_{3}\right]+a\left[\alpha_{4} \alpha_{5}\right]$. Note that $\eta_{2}$ is idempotent:

$$
\eta_{2}^{2}=\left(\left|h_{1}\right\rangle\left\langle h_{1}\right|\right)^{2}=\eta_{2} \quad \text { because } \quad\left\langle h_{1} \mid h_{1}\right\rangle=1 .
$$

\section{PRELIMINARY DISCUSSION OF ENTANGLEMENT IN $\wedge^{3} \mathcal{H}_{6}$}

We consider two approaches for obtaining six-rank approximations for lithium-like ions. One is to work in a scheme of fully restricted spin orbitals. Then the sixth molecular orbital is chosen as $\psi_{3}^{p} \uparrow$. An alternative is to include $\delta_{3} \downarrow$ instead.

For convenience, we use the notation

$$
\left(\begin{array}{c}
|1\rangle \\
|2\rangle \\
|3\rangle \\
|4\rangle \\
|5\rangle \\
|6\rangle
\end{array}\right)=R\left(\begin{array}{c}
\delta_{1} \uparrow \\
\delta_{1} \downarrow \\
\psi_{3}^{p} \downarrow \\
\delta_{2} \downarrow \\
\delta_{2} \uparrow \\
\psi_{3}^{p} \uparrow
\end{array}\right), \quad\left(\begin{array}{c}
|1\rangle \\
|2\rangle \\
|3\rangle \\
|4\rangle \\
|5\rangle \\
|6\rangle
\end{array}\right)=R\left(\begin{array}{c}
\delta_{1} \uparrow \\
\delta_{1} \downarrow \\
\psi_{3}^{p} \downarrow \\
\delta_{2} \downarrow \\
\delta_{2} \uparrow \\
\delta_{3} \downarrow
\end{array}\right),
$$

respectively, for the two cases.

Before discussing the two approaches in detail in the next section, it is useful to first discuss the relations between chemistry and entanglement in each case, in the light of [6] and of the quite recent analysis of universal subspaces for fermionic systems [11]. Without doubt, the search for an entanglement measure for multipartite systems is among the most important challenges facing quantum information theory [12]. For three fermions, there have been some attempts to generalize the Schmidt decomposition, widely used in bipartite systems. Both $[6,11]$ focus on rank 6 descriptions, since these are the lowest nontrivial ones for tripartite systems.

Neither of the choices made in those papers is well adapted to the needs of chemistry, the first being too general and the second too restrictive. The measure of entanglement proposed in [6] on the basis of cubic Jordan algebra theory does not take account of spin partitioning. A wave function $|\Phi\rangle$ belonging to the abstract 20-dimensional Hilbert space $\wedge^{3} \mathcal{H}_{6}$ is considered. Given an ordered basis of $\wedge^{3} \mathcal{H}_{6}$ and

$$
|\Phi\rangle=\sum_{1 \leqslant i<j<k \leqslant 6} c_{i j k}[i j k],
$$

its amount of entanglement is analyzed in terms of the absolute value of the expression

$$
\begin{aligned}
\mathcal{T}:= & 4\left\{\left[\operatorname{Tr}\left(M_{1} M_{2}\right)-\mu \nu\right]^{2}-4 \operatorname{Tr}\left(M_{1}^{\#} M_{2}^{\#}\right)+4 \mu \operatorname{det} M_{1}\right. \\
& \left.+4 \nu \operatorname{det} M_{2}\right\} \quad \text { with } \quad 0 \leqslant|\mathcal{T}| \leqslant 1,
\end{aligned}
$$

where the 20 amplitudes of (8) are arranged in two $3 \times 3$ matrices and two scalars,

$\begin{aligned} M_{1} & :=\left(\begin{array}{lll}c_{156} & -c_{146} & c_{145} \\ c_{256} & -c_{246} & c_{245} \\ c_{356} & -c_{346} & c_{345}\end{array}\right), \\ M_{2} & :=\left(\begin{array}{lll}c_{234} & -c_{134} & c_{124} \\ c_{235} & -c_{135} & c_{125} \\ c_{236} & -c_{136} & c_{126}\end{array}\right), \mu:=c_{123} \text { and } v:=c_{456} .\end{aligned}$

Here, $M^{\#}$ denotes the adjugate of a matrix $M$, such that $M M^{\#}=M^{\#} M=(\operatorname{det} M) I$. Under this measure, nontrivial tripartite entanglement can take place in two inequivalent ways - those with $|\mathcal{T}| \neq 0$ and those with $|\mathcal{T}|=0$-provided that then the pertinent dual wave function $\tilde{\Phi}$ is different from 0. Although both cases exhibit genuine tripartite entanglement (they are neither separable nor biseparable), there is no unitary transformation relating the two types of states. The lowest configuration of the energy with the basis set $\left\{\psi_{3}^{p} \downarrow, \psi_{3}^{p} \uparrow, \delta_{1} \uparrow, \delta_{1} \downarrow, \delta_{2} \uparrow, \delta_{2} \downarrow\right\}$ considered in the first part of this chapter results in a $\mathcal{T}$ measure of entanglement equal to $\mathbf{0}$. In contrast, the wave function constructed from $\left\{\psi_{3}^{p} \downarrow, \delta_{1} \uparrow, \delta_{1} \downarrow, \delta_{2} \uparrow, \delta_{2} \downarrow, \delta_{3} \downarrow\right\}$ results in $\mathcal{T}$ entanglement equal to $2.57 \times 10^{-6}$ (admittedly small, due to quasipinning, as we explain later), which, in particular, means that entanglementwise pinned states and unpinned ones are mutually disconnected.

On the other hand, the framework of the analysis in [11] is applicable for only the first of the two configurations mentioned in (7).

\section{RANK 6 APPROXIMATIONS}

\section{A. Choosing two configurations}

It is readily seen that for the first basis set in (7), of $20=$ $\left(\begin{array}{l}6 \\ 3\end{array}\right)$ Slater determinants there are 9 eigenfunctions of $S_{z}$ with 
TABLE II. Variational energy of Li in a CI picture for different approximation ranks.

\begin{tabular}{lccc}
\hline \hline Rank & Energy & $\alpha$ & $\gamma$ \\
\hline 3 & -7.417919 & 2.686435 & 1.274552 \\
5 & -7.431181 & 2.711177 & 1.304903 \\
$6^{a}$ & -7.431827 & 2.674424 & 1.319161 \\
$6^{b}$ & -7.431639 & 2.712166 & 1.323417 \\
7 & -7.445443 & 2.772402 & 1.336274 \\
8 & -7.454889 & 2.767562 & 1.331108 \\
\hline \hline
\end{tabular}

eigenvalue $\downarrow$ :

[123], [124], [245], [345], [236], [346],

[134], [246], [235].
The first six Slater determinants are eigenvectors of $S^{2}$, which also is true for the combinations

$$
[134]+[246] \text { and }[235]-[134] \text {. }
$$

Consider thus the following wave functions:

$$
\begin{aligned}
& A[123]+B([235]-[134])+E[124]+F[245]+D[345] \\
& \quad+G[236]+H[346]+I([246]+[134]) .
\end{aligned}
$$

The notation corresponds to that of (5), with, however, a numbering change. It is easy to see that the corresponding one-body matrix has the spin structure

$$
\rho_{1}=\rho_{1}^{\uparrow} \oplus \rho_{1}^{\downarrow},
$$

whereby, with respective indices $\{1,5,6\}$ and $\{2,3,4\}$,

$$
\begin{aligned}
& \rho_{1}^{\uparrow}=\left(\begin{array}{ccc}
|A|^{2}+|B|^{2}+|E|^{2}+|I|^{2} & \star & \star \\
\star & |B|^{2}+|D|^{2}+|F|^{2} & \star \\
\star & \star & |G|^{2}+|H|^{2}+|I|^{2}
\end{array}\right), \quad \operatorname{Tr} \rho_{1}^{\uparrow}=1 ; \\
& \rho_{1}^{\downarrow}=\left(\begin{array}{ccc}
|A|^{2}+|B|^{2}+|E|^{2}+|F|^{2}+|G|^{2}+|I|^{2} & \star & \star \\
\star & |A|^{2}+2|B|^{2}+|D|^{2}+|G|^{2}+|H|^{2}+|I|^{2} & \star \\
\star & \star &
\end{array}\right),
\end{aligned}
$$

For the second basis system in (7), among the 20 Slater determinants there are now 12 eigenfunctions of the operator $S^{z}$ with eigenvalue $\downarrow$, namely,

$$
\begin{aligned}
& \text { [123], [124], [245], [345], [134], [235], } \\
& \text { [146], [256], [136], [356], [126], [456]. }
\end{aligned}
$$

Here, we do not write explicitly the general wave function that can be constructed from these and that does not contain any spin contamination.

Table II presents the results for the energy and screening parameters, with $6^{a}$ and $6^{b}$, respectively, denoting the first and second case in (7). In the table we also include the results for higher rank approximations. ${ }^{2}$

Table III gives the results for the natural orbital occupancy numbers.

The (four) Klyachko inequalities for a three-electron system in a rank 6 configuration read

$$
\begin{aligned}
\lambda_{1}+\lambda_{6} & \leqslant 1, \quad \lambda_{2}+\lambda_{5} \leqslant 1, \quad \lambda_{3}+\lambda_{4} \leqslant 1 ; \\
0 & \leqslant \mathcal{D}:=\lambda_{5}+\lambda_{6}-\lambda_{4} .
\end{aligned}
$$

However, one must have $\sum_{i=1}^{6} \lambda_{i}=3$. As a consequence of this, the first inequalities become saturated (the BorlandDennis identities), and there is only one inequality left for further examination. Note that we can formulate this as

$$
\lambda_{1}+\lambda_{2} \leqslant 1+\lambda_{3} \text {. }
$$

\footnotetext{
${ }^{2}$ With our method it is necessary to reach rank 7 in order to obtain part of the (radial) correlation energy. It is well known that the best $\mathrm{HF}$ ground state for Li is given by an unrestricted determinant.
}

Before analyzing $\mathcal{D}$, which is the main subject in this subsection, we emphasize that the Borland-Dennis identities are fulfilled within our numerical accuracy. Also, they imply that in the natural orbital basis every Slater determinant is composed of three orbitals $\left[\alpha_{i} \alpha_{j} \alpha_{k}\right]$, each belonging to one of three different sets, say

$$
\alpha_{i} \in\left\{\alpha_{1}, \alpha_{6}\right\}, \quad \alpha_{j} \in\left\{\alpha_{2}, \alpha_{5}\right\}, \quad \text { and } \quad \alpha_{k} \in\left\{\alpha_{3}, \alpha_{4}\right\} ;
$$

that is, $\wedge^{3} \mathcal{H}_{6}$ splits with a section equal to $\mathcal{H}_{2}^{\otimes 3}$ for a system of three fermions with spin.

Quasipinning is the property of $\mathcal{D}$ being extremely close to 0 . Within our calculation $6^{b}$, we find

$$
0 \leqslant \mathcal{D}=\lambda_{5}+\lambda_{6}-\lambda_{4}=2.1465 \times 10^{-5} .
$$

This value of $\mathcal{D}$ is slightly smaller than the lowest occupation number, $\mathcal{D} / \lambda_{6} \approx 0.97$. D cannot exceed $\lambda_{6}$, because otherwise $\lambda_{5}>\lambda_{4}$. More remarkable is that for the restricted determinant case $6^{a}$, one has $\mathcal{D}$ of order $10^{-12}$, i.e., 0 within numerical accuracy.

Inequality (11) together with the decreasing ordering rule defines a polytope (Fig. 1) in the space of the occupancy numbers.

So far, a number of findings and conclusions can be emphasized:

(a) The energy computed via the restricted basis set $6^{a}$ is (marginally) better than that obtained via $6^{b}$.

(b) Quasipinning is "strict" for $6^{a}$-in fact we do have pinning - and "lax" for $6^{b}$. Indeed, Eq. (12) is still remarkable in absolute terms. But it just means that if the system is close to a vertex, it is close to a face. 
TABLE III. Occupation numbers from ranks 5 to 8 for lithium wave functions.

\begin{tabular}{lccccccr}
\hline \hline Rank & $\lambda_{1}$ & $\lambda_{2}$ & $\lambda_{3}$ & $\lambda_{4} \times 10^{3}$ & $\lambda_{5} \times 10^{3}$ & $\lambda_{6} \times 10^{4}$ & $\lambda_{7} \times 10^{5}$ \\
\hline 5 & 1 & 0.998702 & 0.998702 & 1.297058 & 1.297058 & - & - \\
$6^{a}$ & 0.999978 & 0.998677 & 0.998655 & 1.344195 & 1.322335 & 0.2185 & - \\
$6^{b}$ & 0.999977 & 0.998715 & 0.998715 & 1.284753 & 1.284182 & 0.2203 & - \\
7 & 0.999868 & 0.998629 & 0.998511 & 1.416148 & 1.364978 & 1.2336 & 8.5241 \\
8 & 0.999839 & 0.998663 & 0.998522 & 1.409339 & 1.337846 & 1.3972 & 8.6559 \\
\hline \hline
\end{tabular}

(c) Both states are truly entangled-neither separable nor biseparable. However, the $\mathcal{T}$ measure of entanglement vanishes for $6^{a}$, while $\mathcal{T} \neq 0$ for $6^{b}$. Thus, in some sense the latter is "more entangled" than the former. In fact, referring to the original notation (9), for the case $6^{a}$ we have the expressions

$$
\begin{aligned}
M_{1} & =\left(\begin{array}{ccc}
0 & 0 & 0 \\
0 & -c_{246} & c_{245} \\
0 & -c_{346} & c_{345}
\end{array}\right), \quad M_{2}=\left(\begin{array}{ccc}
0 & -c_{134} & c_{124} \\
c_{235} & 0 & 0 \\
c_{236} & 0 & 0
\end{array}\right), \\
\mu & =c_{123} \text { and } v=0,
\end{aligned}
$$

and hence

$$
\begin{aligned}
\mathcal{T}= & 4\left\{\left[\operatorname{Tr}\left(M_{1} M_{2}\right)-\mu \nu\right]^{2}-4 \operatorname{Tr}\left(M_{1}^{\#} M_{2}^{\#}\right)\right. \\
& \left.+4 \mu \operatorname{det} M_{1}+4 v \operatorname{det} M_{2}\right\}=0
\end{aligned}
$$

For the case $6^{b}$, again referring to the original notation, (9), we deal with

$$
\begin{aligned}
M_{1} & =\left(\begin{array}{lll}
0 & -c_{146} & 0 \\
c_{256} & 0 & c_{245} \\
0 & 0 & c_{345}
\end{array}\right), \quad M_{2}=\left(\begin{array}{lcc}
0 & -c_{134} & c_{124} \\
c_{235} & 0 & 0 \\
0 & 0 & c_{126}
\end{array}\right), \\
\mu & =c_{123} \text { and } v=c_{456},
\end{aligned}
$$

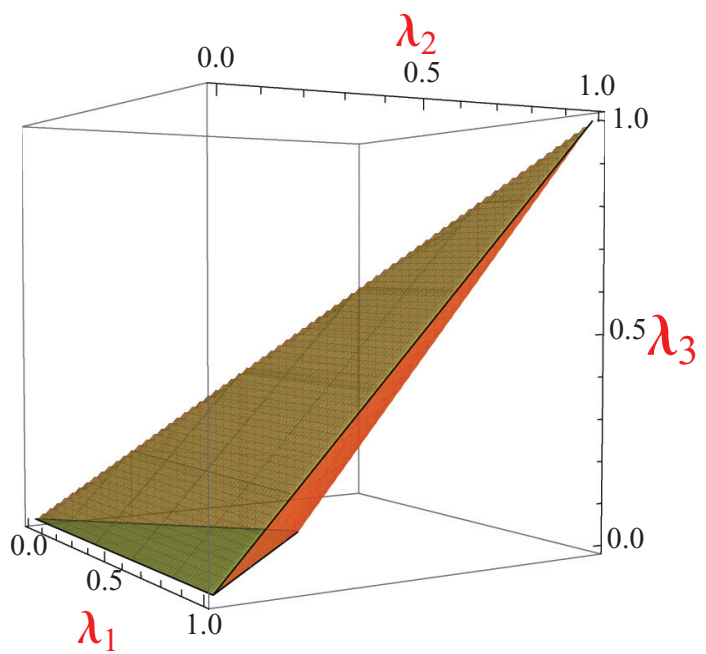

FIG. 1. (Color online) Polytope defined by the expression $\lambda_{1}+$ $\lambda_{2} \leqslant 1+\lambda_{3}$, subject to the condition $1 \geqslant \lambda_{1} \geqslant \lambda_{2} \geqslant \lambda_{3} \geqslant 0$. The saturation condition $\lambda_{1}+\lambda_{2}=1+\lambda_{3}$ is satisfied by the points on one of the faces of the polytope, the one with edges $\lambda_{2}=\lambda_{3}$ for $\lambda_{1}=1$ and $\lambda_{1}=1-\lambda_{2}$ for $\lambda_{3}=0$. The single-determinant state is placed at the corner $\lambda_{1}=\lambda_{2}=\lambda_{3}=1$ of the polytope. The physical ground states appear to be (close to) saturated. and hence

$$
\begin{aligned}
\mathcal{T}= & 4\left\{\left[\operatorname{Tr}\left(M_{1} M_{2}\right)-\mu \nu\right]^{2}-4 \operatorname{Tr}\left(M_{1}^{\#} M_{2}^{\#}\right)\right. \\
& \left.+4 \mu \operatorname{det} M_{1}+4 \nu \operatorname{det} M_{2}\right\} \\
= & 4\left\{\left(-c_{146} c_{235}-c_{134} c_{256}+c_{126} c_{345}-c_{123} c_{456}\right)^{2}\right. \\
& -4\left(c_{134} c_{146} c_{235} c_{256}-c_{126} c_{146} c_{235} c_{245}\right. \\
& \left.-c_{126} c_{134} c_{256} c_{345}\right)+4 c_{123} c_{146} c_{256} c_{345} \\
& \left.+4 c_{456} c_{126} c_{134} c_{235}\right\}=-2.5718 \times 10^{-6} .
\end{aligned}
$$

(d) It is accordingly natural to conjecture, as done in [5], that pinning leads to qualitative differences in multipartite entanglement, and quasipinning correlates negatively with entanglement.

(e) Computing entanglement by means of the standard Jaynes entropy, $-\sum_{i} \lambda_{i} \ln \lambda_{i}$, we obtain $2.05 \times 10^{-2}$ for the restricted configuration and $1.99 \times 10^{-2}$ for the partially unrestricted one. Admittedly, these two values are close, but nevertheless, it would seem to contrarily indicate that $6^{b}$ is "less entangled" than $6^{a}$. In total, this suggests that there is a need to identify genuine multipartite measures of entanglement. A recent proposal [13] looks enticing in this respect.

(f) When the fourth inequality saturates $(\mathcal{D}=0)$, a strong selection rule like (6) applies, namely, the number of Slater determinants reduces to three:

$$
|\Psi\rangle_{3,6}=a\left[\alpha_{1} \alpha_{2} \alpha_{3}\right]+b\left[\alpha_{1} \alpha_{4} \alpha_{5}\right]+c\left[\alpha_{2} \alpha_{4} \alpha_{6}\right] .
$$

It should be clear that $\left\{\alpha_{1}, \alpha_{2}, \alpha_{4}\right\}$ and $\left\{\alpha_{3}, \alpha_{5}, \alpha_{6}\right\}$, respectively, span the spaces on which $\rho_{1}^{\uparrow}$ and $\rho_{1}^{\downarrow}$ in (10) act. The natural occupation numbers for this wave function are of the form

$$
\begin{array}{ll}
\lambda_{1}=|a|^{2}+|b|^{2}, & \lambda_{2}=|a|^{2}+|c|^{2}, \quad \lambda_{3}=|a|^{2}, \\
\lambda_{4}=|b|^{2}+|c|^{2}, & \lambda_{5}=|b|^{2}, \quad \lambda_{6}=|c|^{2} .
\end{array}
$$

(g) When employing a restricted basis set, there is no loss of information in working with the wave function, (13). Even in the general case, at rank 6 simultaneous variation of orbitals and coefficients is still a tempting proposition for the lithium series, in view of the following. The possible loss of information when projecting the total wave function onto this subspace of pinned states has been computed [14]. Given the wave function $|\Phi\rangle \in \wedge^{3} \mathcal{H}_{6}$, and letting $P$ be the projection operator onto the subspace spanned by the Slater determinants $\left[\alpha_{1} \alpha_{2} \alpha_{3}\right],\left[\alpha_{1} \alpha_{4} \alpha_{5}\right]$, and $\left[\alpha_{2} \alpha_{4} \alpha_{6}\right]$, we have the following upper and lower bounds for this projection,

$$
\begin{aligned}
1-\frac{1+2 \xi}{1-4 \xi} \mathcal{D} & \leqslant\|P \Phi\|_{2}^{2} \leqslant 1-\frac{1}{2} \mathcal{D}, \text { provided } \\
\xi & :=3-\lambda_{1}-\lambda_{2}-\lambda_{3}<\frac{1}{4} .
\end{aligned}
$$


Within our calculations the lower bound is larger than $99.997 \%$. Presumably, by dint of astute variation tactics one could obtain extremely good values for the energy with just three Slater terms.

(h) Finally, returning to Ref. [11], the authors there correctly argue that their treatment of universal subspaces gives an alternative proof for the Klyachko representability conditions on $\wedge^{3} \mathcal{H}_{6}$. Conversely, the above gives an independent proof of the assertions in [11], for the same case.

\section{B. Reduced matrices on pinned $\wedge^{3} \mathcal{H}_{6}$}

As in the rank 5 case, the one-body and one-hole matrices read $\rho_{1}=\sum_{i=1}^{6} \lambda_{i}\left|\alpha_{i}\right\rangle\left\langle\alpha_{i}\right|$ and $\eta_{1}=\sum_{i=1}^{6}\left(1-\lambda_{i}\right)\left|\alpha_{i}\right\rangle\left\langle\alpha_{i}\right|$. The two-body and two-hole matrices are, respectively, written as

$$
\begin{aligned}
\rho_{2} & =\sum_{i=1}^{6} \lambda_{i}\left|\omega_{i}\right\rangle\left\langle\omega_{i}\right| \text { and } \eta_{2}=\sum_{i=1}^{6} \mu_{i}\left|h_{i}\right\rangle\left\langle h_{i}\right| \\
& =\sum_{i \in\{1,2,4\}} \lambda_{j}\left|h_{j}\right\rangle\left\langle h_{j}\right|,
\end{aligned}
$$

with $\left|\omega_{i}\right\rangle:=\frac{3}{\sqrt{\lambda_{i}}}\left\langle\alpha_{i} \mid \Psi\right\rangle$, and

$$
\begin{aligned}
\left|h_{1}\right\rangle & =\frac{1}{\sqrt{\lambda_{1}}}\left(b\left[\alpha_{2} \alpha_{3}\right]+a\left[\alpha_{4} \alpha_{5}\right]\right), \\
\left|h_{2}\right\rangle & =\frac{1}{\sqrt{\lambda_{2}}}\left(c\left[\alpha_{1} \alpha_{3}\right]+a\left[\alpha_{4} \alpha_{6}\right]\right), \\
\left|h_{4}\right\rangle & =\frac{1}{\sqrt{\lambda_{4}}}\left(c\left[\alpha_{1} \alpha_{5}\right]+b\left[\alpha_{2} \alpha_{6}\right]\right) ;
\end{aligned}
$$

note that $\left|h_{j}\right\rangle=\left|\omega_{j}\right\rangle$ for $i=3,5,6$ correspond to single determinants. Moreover, $\eta_{2}^{2}=\eta_{2}$.

\section{C. $Z$ dependency of the quasipinning}

The dependence of inequality (12) on the atomic number of the nucleus deserves some extra discussion. The first occupation number will grow as the atomic charge in the nucleus increases. Figure 2 features the evolution of the saturation when $Z$ takes values in $\{3, \ldots, 12\}$. The most relevant measure is $\mathcal{D} / \lambda_{6}$, which (mostly) decreases with $Z$. This means that the numerical distance between $\lambda_{5}$ and $\lambda_{4}$ - or between $\lambda_{2}$ and $\lambda_{3}$-is rapidly decreasing with $Z$.

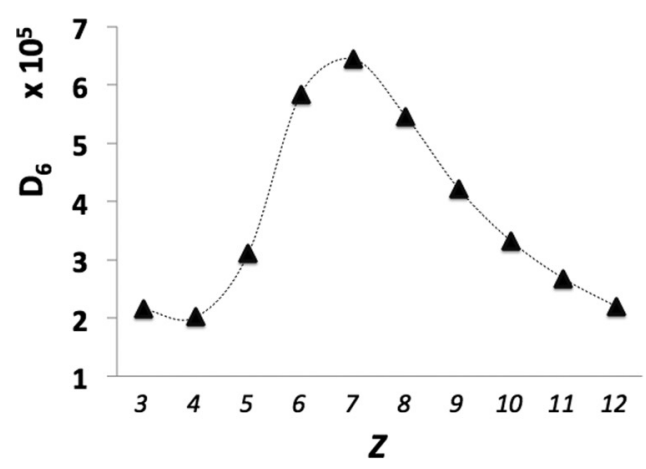

(a)

\section{KLYACHKO THEORY IN HIGHER RANK APPROXIMATIONS}

\section{A. A rank 7 approximation}

We choose the basis set

$$
\left(\begin{array}{c}
|1\rangle \\
|2\rangle \\
|3\rangle \\
|4\rangle \\
|5\rangle \\
|6\rangle \\
|7\rangle
\end{array}\right)=R\left(\begin{array}{c}
\delta_{1} \uparrow \\
\delta_{1} \downarrow \\
\psi_{3}^{p} \downarrow \\
\delta_{2} \downarrow \\
\delta_{2} \uparrow \\
\delta_{3} \downarrow \\
\delta_{3} \uparrow
\end{array}\right) ;
$$

that is, we also include the spin-up counterpart $|7\rangle:=\delta_{3} \uparrow$ of orbital $|6\rangle$. In principle, we have $35=\left(\begin{array}{l}7 \\ 3\end{array}\right)$ Slater determinants, of which 18 have a total $S_{z}$ component equal to $\downarrow$ and 15 are spin adapted: the 8 of the rank 6 approximation $6^{b}$ plus

$$
\begin{aligned}
& \text { [267], [367], [567], [136] - [237], [346] - [357], [257] } \\
& \quad-[156],[257]-[246] .
\end{aligned}
$$

There are four Klyachko inequalities for $\mathrm{Li}$ in a rank 7 configuration:

$$
\begin{array}{ll}
\lambda_{1}+\lambda_{2}+\lambda_{4}+\lambda_{7} \leqslant 2, & \lambda_{1}+\lambda_{2}+\lambda_{5}+\lambda_{6} \leqslant 2, \\
\lambda_{2}+\lambda_{3}+\lambda_{4}+\lambda_{5} \leqslant 2, & \lambda_{1}+\lambda_{3}+\lambda_{4}+\lambda_{6} \leqslant 2 .
\end{array}
$$

In our calculations we find

$$
\begin{aligned}
& 0 \leqslant \mathcal{D}_{7}^{1}=2-\left(\lambda_{1}+\lambda_{2}+\lambda_{4}+\lambda_{7}\right)=0, \\
& 0 \leqslant \mathcal{D}_{7}^{2}=2-\left(\lambda_{1}+\lambda_{2}+\lambda_{5}+\lambda_{6}\right)=1.3045 \times 10^{-5}, \\
& 0 \leqslant \mathcal{D}_{7}^{3}=2-\left(\lambda_{2}+\lambda_{3}+\lambda_{4}+\lambda_{5}\right)=7.7411 \times 10^{-5}, \\
& 0 \leqslant \mathcal{D}_{7}^{4}=2-\left(\lambda_{1}+\lambda_{3}+\lambda_{4}+\lambda_{6}\right)=8.0025 \times 10^{-5} .
\end{aligned}
$$

There are a number of interesting issues regarding the structure of the constraints, which are now briefly discussed.

(a) The pinned system can be factorized:

$$
\wedge^{3} \mathcal{H}_{7} \rightarrow \mathcal{H}_{3} \otimes \wedge^{2} \mathcal{H}_{4}
$$

That is, the one-body matrix can be split into a direct sum of two matrices:

$$
\rho_{1}=\rho_{1}^{\uparrow} \oplus \rho_{1}^{\downarrow} .
$$

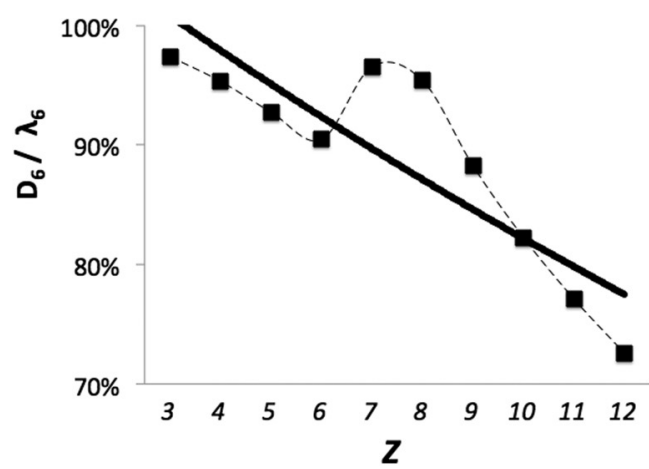

(b)

FIG. 2. (a) $\mathcal{D}$ and (b) $\mathcal{D} / \lambda_{6}$ as functions of the atomic number $Z$ for three-electron systems. 
The first one is a $3 \times 3$ square matrix whose trace is equal to 1 and that is associated with the electron with spin pointing $\uparrow$, while the second matrix is a $4 \times 4$ square matrix whose trace is equal to 2 and is associated with the two electrons with spin pointing $\downarrow$. With the numbering already dictated by the occupancies, its entries read

$$
\begin{aligned}
& \rho_{1}^{\uparrow}(1,1)=\left|c_{123}\right|^{2}+\left|c_{124}\right|^{2}+\left|c_{126}\right|^{2}+\left|c_{134}\right|^{2}+\left|c_{146}\right|^{2}+\left|c_{136}\right|^{2}, \\
& \rho_{1}^{\uparrow}(1,2)=c_{123} c_{235}^{*}+c_{124} c_{245}^{*}-c_{126} c_{256}^{*}+c_{134} c_{345}^{*}-c_{136} c_{356}^{*}-c_{146} c_{456}^{*}, \\
& \rho_{1}^{\uparrow}(1,3)=c_{123} c_{237}^{*}+c_{124} c_{247}^{*}+c_{126} c_{267}^{*}+c_{134} c_{347}^{*}+c_{136} c_{367}^{*}+c_{146} c_{467}^{*}, \\
& \rho_{1}^{\uparrow}(2,2)=\left|c_{235}\right|^{2}+\left|c_{245}\right|^{2}+\left|c_{256}\right|^{2}+\left|c_{345}\right|^{2}+\left|c_{356}\right|^{2}+c_{456}, \\
& \rho_{1}^{\uparrow}(2,3)=c_{235} c_{237}^{*}+c_{245} c_{247}^{*}-c_{256} c_{267}^{*}+c_{345} c_{347}^{*}-c_{356} c_{367}^{*}-c_{456} c_{467}^{*}, \\
& \rho_{1}^{\uparrow}(3,3)=\left|c_{237}\right|^{2}+\left|c_{247}\right|^{2}+\left|c_{267}\right|^{2}+\left|c_{347}\right|^{2}+\left|c_{367}\right|^{2}+c_{467}
\end{aligned}
$$

and

$$
\begin{aligned}
& \rho_{1}^{\downarrow}(1,1)=\left|c_{123}\right|^{2}+\left|c_{124}\right|^{2}+\left|c_{126}\right|^{2}+\left|c_{235}\right|^{2}+\left|c_{237}\right|^{2}+\left|c_{245}\right|^{2}+\left|c_{2 d n 47}\right|^{2}+\left|c_{256}\right|^{2}+\left|c_{267}\right|^{2}, \\
& \rho_{1}^{\downarrow}(1,2)=c_{124} c_{134}^{*}+c_{126} c_{136}^{*}+c_{245} c_{345}^{*}+c_{247} c_{347}^{*}+c_{256} c_{356}^{*}+c_{267} c_{367}^{*}, \\
& \rho_{1}^{\downarrow}(1,3)=-c_{123} c_{134}^{*}+c_{126} c_{146}^{*}-c_{235} c_{345}^{*}-c_{237} c_{347}^{*}+c_{256} c_{456}^{*}+c_{267} c_{467}^{*}, \\
& \rho_{1}^{\downarrow}(1,4)=-c_{123} c_{136}^{*}-c_{124} c_{146}^{*}+c_{235} c_{356}^{*}-c_{237} c_{367}^{*}+c_{245} c_{456}^{*}-c_{247} c_{467}^{*}, \\
& \rho_{1}^{\downarrow}(2,2)=\left|c_{123}\right|^{2}+\left|c_{134}\right|^{2}+\left|c_{136}\right|^{2}+\left|c_{235}\right|^{2}+\left|c_{237}\right|^{2}+\left|c_{345}\right|^{2}+\left|c_{347}\right|^{2}+\left|c_{356}\right|^{2}+\left|c_{367}\right|^{2}, \\
& \rho_{1}^{\downarrow}(2,3)=c_{123} c_{124}^{*}+c_{136} c_{146}^{*}+c_{235} c_{245}^{*}+c_{237} c_{247}^{*}-c_{356} c_{456}^{*}+c_{367} c_{467}^{*}, \\
& \rho_{1}^{\downarrow}(2,4)=c_{123} c_{126}^{*}-c_{134} c_{146}^{*}-c_{235} c_{256}^{*}+c_{237} c_{267}^{*}+c_{345} c_{456}^{*}-c_{347} c_{467}^{*}, \\
& \rho_{1}^{\downarrow}(3,3)=\left|c_{124}\right|^{2}+\left|c_{134}\right|^{2}+\left|c_{146}\right|^{2}+\left|c_{245}\right|^{2}+\left|c_{247}\right|^{2}+\left|c_{345}\right|^{2}+\left|c_{347}\right|^{2}+\left|c_{456}\right|^{2}+\left|c_{467}\right|^{2}, \\
& \rho_{1}^{\downarrow}(3,4)=c_{124} c_{126}^{*}+c_{134} c_{136}^{*}-c_{245} c_{256}^{*}+c_{247} c_{267}^{*}-c_{345} c_{356}^{*}+c_{347} c_{367}^{*}, \\
& \rho_{1}^{\downarrow}(4,4)=\left|c_{126}\right|^{2}+\left|c_{136}\right|^{2}+\left|c_{146}\right|^{2}+\left|c_{256}\right|^{2}+\left|c_{267}\right|^{2}+\left|c_{356}\right|^{2}+\left|c_{367}\right|^{2}+\left|c_{456}\right|^{2}+\left|c_{467}\right|^{2} .
\end{aligned}
$$

(b) For the first time we see the appearance of two scales of quasipinning.

(c) If the second constraint is saturated, the selection rule fixes the number of Slater determinants in the decomposition of the wave function to be nine:

$\left[\alpha_{1} \alpha_{2} \alpha_{3}\right],\left[\alpha_{1} \alpha_{4} \alpha_{5}\right],\left[\alpha_{1} \alpha_{4} \alpha_{6}\right],\left[\alpha_{1} \alpha_{5} \alpha_{7}\right],\left[\alpha_{1} \alpha_{6} \alpha_{7}\right]$,

$\left[\alpha_{2} \alpha_{4} \alpha_{5}\right],\left[\alpha_{2} \alpha_{4} \alpha_{6}\right],\left[\alpha_{2} \alpha_{5} \alpha_{7}\right],\left[\alpha_{2} \alpha_{6} \alpha_{7}\right]$.

(d) As for the case of $\wedge^{3} \mathcal{H}_{6}$, the loss of information when projecting the total wave function onto this ninedimensional subspace of twice-pinned states can be estimated. In Appendix B we give a proof of the following theorem: let a wave function $|\Phi\rangle \in \bigwedge^{3} \mathcal{H}_{7}$ with natural orbitals $\left|\alpha_{i}\right\rangle_{i=1}^{7}$, occupation numbers $\left\{\lambda_{i}\right\}_{i=1}^{7}$, saturating the first restriction. Moreover, let $P_{7}$ be the projection operator onto the subspace spanned by the Slater determinants above. Then the upper and lower bounds of this projection are given by

$$
\begin{aligned}
& 1-\frac{1+9 \xi}{1-11 \xi} \mathcal{D}_{7}^{2} \leqslant\left\|P_{7} \Phi\right\|_{2}^{2} \leqslant 1-\frac{1}{2} \mathcal{D}_{7}^{2} \\
& \text { provided that } \xi<\frac{1}{11} .
\end{aligned}
$$

Within our calculations, $\quad 1-\frac{1+9 \xi}{1-11 \xi} \mathcal{D}_{7}^{2}=1-1.3852 \times$ $10^{-5}=99.9986 \%$. (e) If, in addition, the third or the fourth constraint becomes saturated, the selection rules decreases the number of allowed determinants to just 4 . Saturating both simultaneously reduces the case to the saturated rank 6 wave function.

We omit the expressions of the two-body and two-hole matrices, which can be easily calculated. It should, however, be added that the tensor character under rotations of the reduced matrices for a three-electron system is quite different from the one for a two-electron system; in particular, the relative weight in the lithium isoelectronic series of the six components identified in Sec. 6A of [15] or in [16] deserves some further study.

\section{B. Quasipinning displayed in the rank 8 approximation}

We can obtain rank 8 by adding a new orbital $|8\rangle:=\delta_{4} \downarrow$, giving now $\left(\begin{array}{l}3 \\ 1\end{array}\right)\left(\begin{array}{l}5 \\ 2\end{array}\right)=30$ Slater determinants with the correct $z$ component of the spin. Among them, 21 are spin adapted, i.e., the 15 of the rank 7 approximation, plus

$$
\begin{gathered}
\text { [128], [458], [678], [148] - [258], } \\
{[168]-[278],[568]-[478] .}
\end{gathered}
$$

The number of Klyachko inequalities grows notably with the rank. We find 31 inequalities in [3]. Of those, 28 constraints are displayed in Table IV here. 
TABLE IV. Klyachko inequalities for a system $\wedge^{3} \mathcal{H}_{8}$ and some numerical values for $\mathrm{Li}$.

\begin{tabular}{|c|c|}
\hline Inequality & Value $\times 10^{3}$ \\
\hline $\begin{array}{l}\mathcal{D}_{8}^{1}=2-\left(\lambda_{1}+\lambda_{2}+\lambda_{4}+\lambda_{7}\right) \\
\mathcal{D}_{8}^{2}=2-\left(\lambda_{1}+\lambda_{2}+\lambda_{5}+\lambda_{6}\right) \\
\mathcal{D}_{8}^{3}=2-\left(\lambda_{2}+\lambda_{3}+\lambda_{4}+\lambda_{5}\right) \\
\mathcal{D}_{8}^{4}=2-\left(\lambda_{1}+\lambda_{3}+\lambda_{4}+\lambda_{6}\right)\end{array}$ & $\begin{array}{l}0.0017 \\
0.0200 \\
0.0671 \\
0.0894\end{array}$ \\
\hline $\begin{array}{l}0 \leqslant \mathcal{D}_{8}^{5}=1-\left(\lambda_{1}+\lambda_{2}-\lambda_{3}\right) \\
0 \leqslant \mathcal{D}_{8}^{6}=1-\left(\lambda_{2}+\lambda_{5}-\lambda_{7}\right) \\
0 \leqslant \mathcal{D}_{8}^{7}=1-\left(\lambda_{1}+\lambda_{6}-\lambda_{7}\right) \\
0 \leqslant \mathcal{D}_{8}^{8}=1-\left(\lambda_{2}+\lambda_{4}-\lambda_{6}\right) \\
0 \leqslant \mathcal{D}_{8}^{9}=1-\left(\lambda_{1}+\lambda_{4}-\lambda_{5}\right) \\
0 \leqslant \mathcal{D}_{8}^{10}=1-\left(\lambda_{3}+\lambda_{4}-\lambda_{7}\right)\end{array}$ & $\begin{array}{l}0.0200 \\
0.0854 \\
0.1078 \\
0.0671 \\
0.0894 \\
0.1548\end{array}$ \\
\hline $0 \leqslant \mathcal{D}_{8}^{11}=1-\left(\lambda_{1}+\lambda_{8}\right)$ & 0.1592 \\
\hline $\begin{array}{l}0 \leqslant \mathcal{D}_{8}^{12}=-\left(\lambda_{2}-\lambda_{3}-\lambda_{6}-\lambda_{7}\right) \\
0 \leqslant \mathcal{D}_{8}^{13}=-\left(\lambda_{4}-\lambda_{5}-\lambda_{6}-\lambda_{7}\right) \\
0 \leqslant \mathcal{D}_{8}^{14}=-\left(\lambda_{1}-\lambda_{3}-\lambda_{5}-\lambda_{7}\right) \\
0 \leqslant \mathcal{D}_{8}^{15}=2-\left(\lambda_{2}+\lambda_{3}+2 \lambda_{4}-\lambda_{5}-\lambda_{7}+\lambda_{8}\right) \\
0 \leqslant \mathcal{D}_{8}^{16}=2-\left(\lambda_{1}+\lambda_{3}+2 \lambda_{4}-\lambda_{5}-\lambda_{6}+\lambda_{8}\right) \\
0 \leqslant \mathcal{D}_{8}^{17}=2-\left(\lambda_{1}+2 \lambda_{2}-\lambda_{3}+\lambda_{4}-\lambda_{5}+\lambda_{8}\right) \\
0 \leqslant \mathcal{D}_{8}^{18}=2-\left(\lambda_{1}+2 \lambda_{2}-\lambda_{3}+\lambda_{5}-\lambda_{6}+\lambda_{8}\right)\end{array}$ & $\begin{array}{l}0.0854 \\
0.1548 \\
0.1078 \\
1.4183 \\
0.2956 \\
1.2836 \\
0.1569\end{array}$ \\
\hline $0 \leqslant \mathcal{D}_{8}^{19}=-\left(\lambda_{1}+\lambda_{2}-2 \lambda_{3}-\lambda_{4}-\lambda_{5}\right)$ & 1.2897 \\
\hline $0 \leqslant \mathcal{D}_{8}^{21}=-\left(\lambda_{1}-\lambda_{3}-\lambda_{4}-\lambda_{5}+\lambda_{8}\right)$ & 1.4288 \\
\hline $\begin{array}{l}0 \leqslant \mathcal{D}_{8}^{23}=1-\left(2 \lambda_{1}-\lambda_{2}+\lambda_{4}-2 \lambda_{5}-\lambda_{6}+\lambda_{8}\right) \\
0 \leqslant \mathcal{D}_{8}^{24}=1-\left(\lambda_{3}+2 \lambda_{4}-2 \lambda_{5}-\lambda_{6}-\lambda_{7}+\lambda_{8}\right) \\
0 \leqslant \mathcal{D}_{8}^{25}=1-\left(2 \lambda_{1}-\lambda_{2}-\lambda_{4}+\lambda_{6}-2 \lambda_{7}+\lambda_{8}\right) \\
0 \leqslant \mathcal{D}_{8}^{26}=1-\left(2 \lambda_{1}+\lambda_{2}-2 \lambda_{3}-\lambda_{4}-\lambda_{6}+\lambda_{8}\right) \\
0 \leqslant \mathcal{D}_{8}^{27}=1-\left(\lambda_{1}+2 \lambda_{2}-2 \lambda_{3}-\lambda_{5}-\lambda_{6}+\lambda_{8}\right)\end{array}$ & $\begin{array}{l}0.3894 \\
1.5591 \\
0.4262 \\
0.2507 \\
1.3551\end{array}$ \\
\hline $\begin{array}{l}0 \leqslant \mathcal{D}_{8}^{29}=\lambda_{1}-\lambda_{3}-2 \lambda_{4}+3 \lambda_{5}+2 \lambda_{6}+\lambda_{7}-\lambda_{8} \\
0 \leqslant \mathcal{D}_{8}^{30}=-\left(2 \lambda_{1}+\lambda_{2}-3 \lambda_{3}-2 \lambda_{4}-\lambda_{5}-\lambda_{6}+\lambda_{8}\right) \\
0 \leqslant \mathcal{D}_{8}^{31}=-\left(\lambda_{1}+2 \lambda_{2}-3 \lambda_{3}-\lambda_{4}-2 \lambda_{5}-\lambda_{6}+\lambda_{8}\right)\end{array}$ & $\begin{array}{l}2.8758 \\
1.5204 \\
2.6247\end{array}$ \\
\hline
\end{tabular}

In the table, we include the values of the inequalities that result from our calculation, and in order to analyze these further, we plot them both on a linear and on a logarithmic scale in Fig. 3. The presence of several scales is clearly shown. Moreover, conditions involving the eighth occupation number are clearly weaker than the previous ones. The main point, which both confirms and extends the findings for the toy model in [5], is the robustness of quasipinning. In particular, the quantity $\mathcal{D}_{8}^{1}$, found to be exactly 0 in the previous rank, remains in a strongly pinned regime.

Finally, one can examine the effect of the saturation conditions and the resulting dramatic reduction in the number

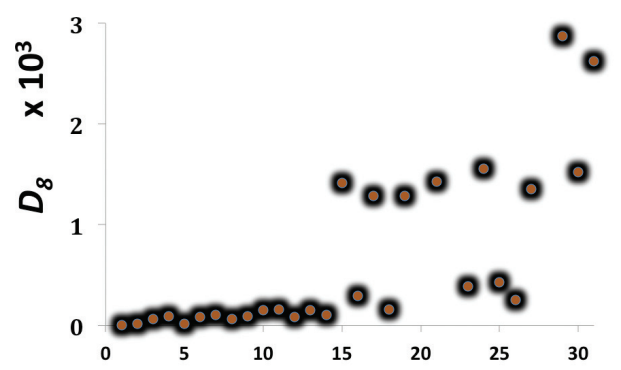

(a)

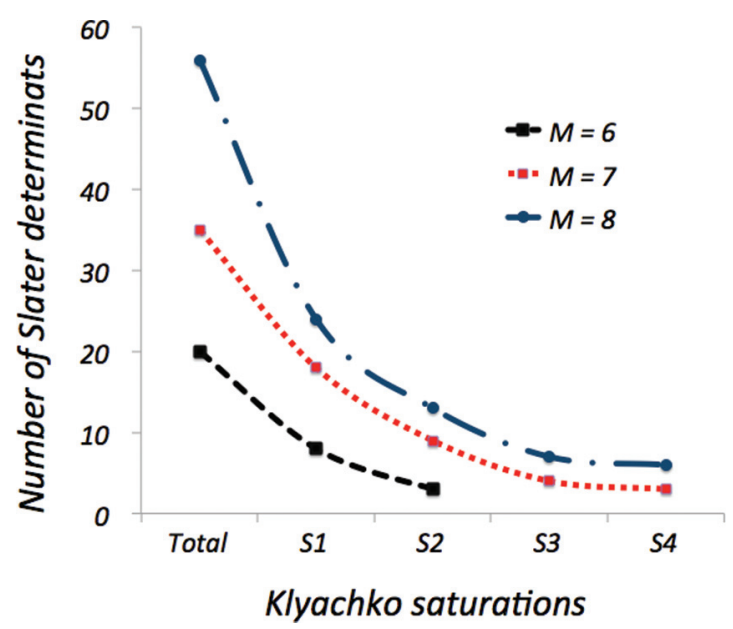

FIG. 4. (Color online) Evolution of the dimension of the space of Slater determinants as a function of Klyachko's saturations for ranks 6,7 , and 8 .

of Slater determinants, as well as the simplification of the corresponding two-body matrix. This remarkable evolution is visualized in Fig. 4.

\section{CONCLUSION}

By means of numerical calculations, we have explored the nature of the quasipinning in real three-electron atoms. In the space $\wedge^{3} \mathcal{H}_{6}$, for restricted spin orbitals we find that the Klyachko constraint is saturated. For unrestricted configurations, quasipinning is bounded by the lowest occupation number $\lambda_{6}$. In approximations of higher rank, the Klyachko constraints split into well-differentiated groups of different levels of saturation. In other words, for a real system we find results compatible with those found previously for the model system [5]. A simple geometric probability argument also suggests stability of quasipinning. Moreover, whenever $3-\lambda_{1}-\lambda_{2}-\lambda_{3}$ is not far from 0 , projecting the complete wave function of the CI picture into the set of pinned states appears to result in a negligible loss of information. Thus, the Klyachko-guided addition of a few Slater determinants to HF-type states becomes a low-cost approach to accurate atomic wave functions.

In addition, through our work we have verified some recent results of quantum information theory for three-electron

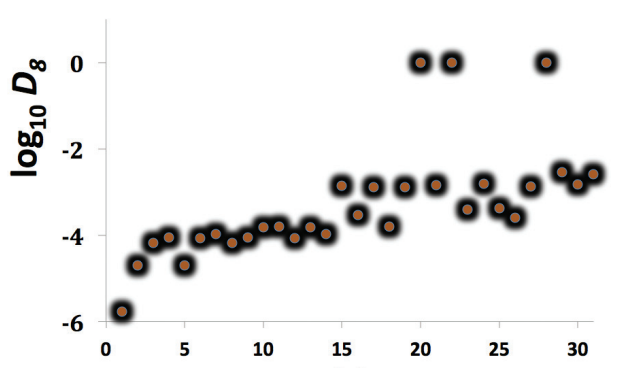

(b)

FIG. 3. (Color online) The value of the quantities $\mathcal{D}_{8}^{j}$ of Table IV as a function of $j$ (a) in values of $10^{-3}$ and (b) on a logarithmic scale. 
systems. In particular, we find nontrivial, but fairly low, quantum entanglement in ground states.

Even if the present study is a step towards a general description of real systems, the system of our study is special in a couple of aspects. The HF approximation is a good starting point for Li-like atoms, and the system has a high spherical symmetry. In future work we hope to address cases where the occupation numbers do not lie so close to 0 or 1 and/or the symmetry is reduced. We will thereby be able to explore whether the conclusions we have drawn in the present study hold more generally in chemistry.

\section{ACKNOWLEDGMENTS}

We are grateful to J. C. Várilly for helpful comments. C. L. B. R. was supported by a Francisco José de Caldas scholarship, funded by Colciencias. He very much appreciates the warm atmosphere of the Physikalische und Theoretische Chemie group at Saarlandes Universität. J.M.G.B. was supported by the Diputación General de Aragón (Grant No. DGIID-DGAE24/2) and the defunct Ministerio de Educación y Ciencia (Grant No. FPA2009-09638).

\section{APPENDIX A: ON THE NATURE OF THE KLYACHKO RESTRICTIONS}

Here, we do not give proofs of the Klyachko constraints but just discuss a few aspects of relevance to the present work. It is useful to consider the skew Cauchy formula,

$$
\bigwedge^{N}\left(\mathcal{H}_{\mathrm{s}} \otimes \mathcal{H}_{\mathrm{orb}}\right)=\sum_{|\kappa|=N} \mathcal{H}_{\mathrm{s}}^{\kappa} \otimes \mathcal{H}_{\mathrm{orb}}^{\bar{\kappa}}
$$

Here, $\kappa$ denotes the representation corresponding to the partition or Young tableau $\kappa$, and $\bar{\kappa}$ is the dual partition.

In the present work we have exclusively the case $\operatorname{dim} \mathcal{H}_{\mathrm{s}}=$ 2 and $N=3$, which makes everything relatively simple. The three-electron state space splits into spin-orbital sectors, which one needs to specify in order to check quasipinning, as well as to gauge entanglement. $\mathcal{H}_{\mathrm{s}}$ corresponds to a spin- $\frac{1}{2}$ particle. Therefore, on the left-hand side we may have only representations of $\mathrm{SU}(2)$; i.e., either $j=\frac{1}{2}$ or $j=\frac{3}{2}$ for three particles. Since there are no skew-symmetric combinations of three spins- $\frac{1}{2}$, the partition $(1,1,1)$ on the right-hand side plays no role; consequently, only tableaux with up to two columns may appear on the left-hand side.

Consider, for instance, the first nontrivial case $\wedge^{3}\left(\mathcal{H}_{2} \otimes\right.$ $\mathcal{H}_{3}$ ) in configuration $6^{a}$ in Sec. IV. There are 20 configurations in all. Clearly there is 1 with three spin down and 1 with three spin up, belonging to the representation with $j=\frac{3}{2}$. Of the 18 remaining states, 9 have one spin down in total, and 9 have spin up. But only 8 of each belong to the $j=\frac{1}{2}$ representation; the other 20 belong to $j=\frac{3}{2}$, whereby the spatial orbitals enter in the unique completely skew-symmetric combination. This takes care of "spin contamination." Accordingly,

$$
\wedge^{3}\left(\mathcal{H}_{2 \mathrm{~s}} \otimes \mathcal{H}_{3 \text { orb }}\right)=\downarrow \mathcal{H}_{2}^{\otimes 3} \oplus \uparrow \mathcal{H}_{2}^{\otimes 3}+\mathcal{H}_{\mathrm{s}}^{3 / 2} \otimes \wedge^{3} \mathcal{H}_{3 \text { orb }}
$$

From these simple observations to the generalized Pauli constraints there is still a long haul, demanding generous dollops of Kirillov's theory of orbits of the coadjoint action for compact groups [3]; the surprising outcome is that only linear inequalities are found.

Of course, not all of our basis sets conform to the left-hand side of (A1). This causes no problem, however, since any basis set can be considered a special case of a larger one with the "right" structure, with some holes. It is important that the Klyachko restrictions are consistent, so lower rank ones can be derived from higher rank ones. Recall, for instance, our example $\wedge^{3} \mathcal{H}_{7}$ and the four corresponding relations:

$$
\begin{array}{ll}
\lambda_{1}+\lambda_{2}+\lambda_{4}+\lambda_{7} \leqslant 2, & \lambda_{1}+\lambda_{2}+\lambda_{5}+\lambda_{6} \leqslant 2, \\
\lambda_{2}+\lambda_{3}+\lambda_{4}+\lambda_{5} \leqslant 2, & \lambda_{1}+\lambda_{3}+\lambda_{4}+\lambda_{6} \leqslant 2 .
\end{array}
$$

At first, the original Pauli principle $\lambda_{1} \leqslant 1$ is perhaps not entirely obvious here; it follows from summing the second and the fourth. Also, let us consider the case $\lambda_{7}=0$. Then summing the second and the third we obtain $\lambda_{2}+\lambda_{5} \leqslant 1$; the second and fourth yield $\lambda_{3}+\lambda_{4} \leqslant 1$, and so on: we plainly recover the Borland-Dennis relations for $\wedge^{3} \mathcal{H}_{6}$. The reader will have no difficulty in retrieving all the lower rank relations from the ones on $\wedge^{3} \mathcal{H}_{8}$.

\section{APPENDIX B: BOUNDS FOR THE RANK 7 APPROXIMATION}

Theorem. Let $|\Phi\rangle \in \wedge^{3} \mathcal{H}_{7}$ be a wave function with natural orbitals $\left\{\left|\alpha_{i}\right\rangle\right\}_{i=1}^{7}$ and occupation numbers $\left\{\lambda_{i}\right\}_{i=1}^{7}$ arranged in decreasing order. Let $P_{7}$ be the projection operator onto the subspace spanned by the Slater determinants:

$\left[\alpha_{1} \alpha_{2} \alpha_{3}\right],\left[\alpha_{1} \alpha_{4} \alpha_{5}\right],\left[\alpha_{1} \alpha_{4} \alpha_{6}\right],\left[\alpha_{1} \alpha_{5} \alpha_{7}\right],\left[\alpha_{1} \alpha_{6} \alpha_{7}\right]$,

$\left[\alpha_{2} \alpha_{4} \alpha_{5}\right],\left[\alpha_{2} \alpha_{4} \alpha_{6}\right],\left[\alpha_{2} \alpha_{5} \alpha_{7}\right],\left[\alpha_{2} \alpha_{6} \alpha_{7}\right]$.

Upper and lower bounds of this projection are given by

$$
\begin{aligned}
1-\frac{1+9 \xi}{1-11 \xi} \mathcal{D}_{7}^{2} & \leqslant\left\|P_{7} \Phi\right\|_{2}^{2} \leqslant 1-\frac{1}{2} \mathcal{D}_{7}^{2}, \\
\text { where } \xi & :=3-\lambda_{1}-\lambda_{2}-\lambda_{3}<\frac{1}{11} .
\end{aligned}
$$

Proof. Let $J_{1}=\{3,5,6\}$ and $J_{2}=\{1,2,4,7\}$. A general wave function in $\wedge^{3} \mathcal{H}_{7}$ is given by

$$
\begin{aligned}
|\Phi\rangle & =\sum_{\substack{k \in J_{1} \\
i, j \in J_{2}}} c_{i j k}\left[\alpha_{i} \alpha_{j} \alpha_{k}\right] \text { and consequently } \\
\lambda_{m} & =\sum_{\substack{k \in J_{1} \\
i, j \in J_{2} \\
m \in\{i, j, k\}}}\left|c_{i j k}\right|^{2} .
\end{aligned}
$$

Therefore,

$$
\begin{aligned}
& \lambda_{1}=\left|c_{123}\right|^{2}+\left|c_{125}\right|^{2}+\left|c_{126}\right|^{2}+\left|c_{134}\right|^{2}+\left|c_{145}\right|^{2}+\left|c_{146}\right|^{2}+\left|c_{137}\right|^{2}+\left|c_{157}\right|^{2}+\left|c_{167}\right|^{2} \\
& \lambda_{2}=\left|c_{123}\right|^{2}+\left|c_{125}\right|^{2}+\left|c_{126}\right|^{2}+\left|c_{234}\right|^{2}+\left|c_{245}\right|^{2}+\left|c_{246}\right|^{2}+\left|c_{237}\right|^{2}+\left|c_{257}\right|^{2}+\left|c_{267}\right|^{2}
\end{aligned}
$$




$$
\begin{aligned}
& \lambda_{5}=\left|c_{125}\right|^{2}+\left|c_{145}\right|^{2}+\left|c_{157}\right|^{2}+\left|c_{245}\right|^{2}+\left|c_{257}\right|^{2}+\left|c_{457}\right|^{2}, \\
& \lambda_{6}=\left|c_{126}\right|^{2}+\left|c_{146}\right|^{2}+\left|c_{167}\right|^{2}+\left|c_{246}\right|^{2}+\left|c_{267}\right|^{2}+\left|c_{467}\right|^{2} .
\end{aligned}
$$

A simple computation gives

$$
\begin{aligned}
\lambda_{1}+\lambda_{2}+\lambda_{5}+\lambda_{6}= & 2\left|c_{123}\right|^{2}+3\left|c_{125}\right|^{2}+3\left|c_{126}\right|^{2}+\left|c_{134}\right|^{2}+2\left|c_{145}\right|^{2}+2\left|c_{146}\right|^{2}+\left|c_{137}\right|^{2} \\
& +2\left|c_{157}\right|^{2}+2\left|c_{167}\right|^{2}+\left|c_{234}\right|^{2}+2\left|c_{245}\right|^{2}+2\left|c_{246}\right|^{2}+\left|c_{237}\right|^{2}+2\left|c_{257}\right|^{2}+2\left|c_{267}\right|^{2}+\left|c_{457}\right|^{2} \\
& +\left|c_{467}\right|^{2} .
\end{aligned}
$$

And trivially,

$$
\mathcal{D}_{7}^{2}=2-\left(\lambda_{1}+\lambda_{2}+\lambda_{5}+\lambda_{6}\right)=L+S-\left|c_{125}\right|^{2}-\left|c_{126}\right|^{2},
$$

$S:=2\left|c_{347}\right|^{2}+\left|c_{457}\right|^{2}+\left|c_{467}\right|^{2}$, and $L:=\left|c_{134}\right|^{2}+\left|c_{137}\right|^{2}+\left|c_{234}\right|^{2}+\left|c_{237}\right|^{2}$. Thus, for the projection onto the aforementioned subspace we have

$$
\begin{aligned}
\left\|P_{7} \Phi\right\|_{2}^{2} & =\left|c_{123}\right|^{2}+\left|c_{145}\right|^{2}+\left|c_{146}\right|^{2}+\left|c_{157}\right|^{2}+\left|c_{167}\right|^{2}+\left|c_{245}\right|^{2}+\left|c_{246}\right|^{2}+\left|c_{257}\right|^{2}+\left|c_{267}\right|^{2} \\
& =1-\left(L+\left|c_{347}\right|^{2}+\left|c_{125}\right|^{2}+\left|c_{457}\right|^{2}+\left|c_{126}\right|^{2}+\left|c_{467}\right|^{2}\right) \\
& \leqslant 1-\frac{1}{2}\left(L+2\left|c_{347}\right|^{2}-\left|c_{125}\right|^{2}+\left|c_{457}\right|^{2}-\left|c_{126}\right|^{2}+\left|c_{467}\right|^{2}\right)=1-\frac{1}{2} \mathcal{D}_{7}^{2},
\end{aligned}
$$

which is the upper bound of (B1).

To establish the lower bound, note that in the basis of natural orbitals we know that

$$
\begin{aligned}
& \left\langle\alpha_{6}\left|\rho_{1}\right| \alpha_{3}\right\rangle=c_{123}^{*} c_{126}-c_{134}^{*} c_{146}+c_{137}^{*} c_{167}-c_{234}^{*} c_{246}+c_{237}^{*} c_{267}-c_{347}^{*} c_{467}=0, \\
& \left\langle\alpha_{5}\left|\rho_{1}\right| \alpha_{3}\right\rangle=c_{123}^{*} c_{125}-c_{134}^{*} c_{145}+c_{137}^{*} c_{157}+c_{234}^{*} c_{245}+c_{237}^{*} c_{257}-c_{347}^{*} c_{457}=0 .
\end{aligned}
$$

Let $\epsilon:=c_{123}$, the amplitude of the HF determinant. Using the Cauchy inequality $(A+B+C+D+E)^{2} \leqslant 5\left(A^{2}+B^{2}+C^{2}+\right.$ $D^{2}+E^{2}$ ) as well as $\left|c_{a b c}\right|^{2} \leqslant 1-|\epsilon|^{2}$ whenever $a b c \neq 123$, we obtain

$$
\begin{aligned}
\left|c_{126}\right|^{2} & \leqslant \frac{5}{|\epsilon|^{2}}\left[\left|c_{134}\right|^{2}\left|c_{146}\right|^{2}+\left|c_{137}\right|^{2}\left|c_{167}\right|^{2}+\left|c_{234}\right|^{2}\left|c_{246}\right|^{2}+\left|c_{237}\right|^{2}\left|c_{267}\right|^{2}+\left|c_{347}\right|^{2}\left|c_{467}\right|^{2}\right] \\
& \leqslant \frac{5\left(1-|\epsilon|^{2}\right)}{|\epsilon|^{2}}\left[L+\frac{1}{2}\left(\left|c_{347}\right|^{2}+\left|c_{467}\right|^{2}\right)\right], \\
\left|c_{125}\right|^{2} & \leqslant \frac{5}{|\epsilon|^{2}}\left[\left|c_{134}\right|^{2}\left|c_{145}\right|^{2}+\left|c_{137}\right|^{2}\left|c_{157}\right|^{2}+\left|c_{234}\right|^{2}\left|c_{245}\right|^{2}+\left|c_{237}\right|^{2}\left|c_{257}\right|^{2}+\left|c_{347}\right|^{2}\left|c_{457}\right|^{2}\right] \\
& \leqslant \frac{5\left(1-|\epsilon|^{2}\right)}{|\epsilon|^{2}}\left[L+\frac{1}{2}\left(\left|c_{347}\right|^{2}+\left|c_{457}\right|^{2}\right)\right] .
\end{aligned}
$$

Let us set, for some $r, u \geqslant 0$,

$$
\begin{aligned}
L+\left|c_{347}\right|^{2}+\left|c_{125}\right|^{2}+\left|c_{457}\right|^{2}+\left|c_{126}\right|^{2}+\left|c_{467}\right|^{2} \leqslant & L+(1+u) S+(1-r)\left(\left|c_{125}\right|^{2}+\left|c_{126}\right|^{2}\right)+r\left(\left|c_{125}\right|^{2}+\left|c_{126}\right|^{2}\right) \\
\leqslant & L+(1+u) S+(1-r)\left(\left|c_{125}\right|^{2}+\left|c_{126}\right|^{2}\right)+\frac{5 r\left(1-|\epsilon|^{2}\right)}{|\epsilon|^{2}}\left[2 L+\frac{1}{2} S\right] \\
= & {\left[1+\frac{10 r\left(1-|\epsilon|^{2}\right)}{|\epsilon|^{2}}\right] L+(1-r)\left(\left|c_{125}\right|^{2}+\left|c_{126}\right|^{2}\right) } \\
& +\left[(1+u)+\frac{5 r\left(1-|\epsilon|^{2}\right)}{2|\epsilon|^{2}}\right] S .
\end{aligned}
$$

By choosing

$$
r=\frac{2|\epsilon|^{2}}{11|\epsilon|^{2}-10} \quad \text { and } \quad u=\frac{15\left(1-|\epsilon|^{2}\right)}{11|\epsilon|^{2}-10} \quad \text { with } \quad|\epsilon|^{2}>10 / 11,
$$

we obtain that $L+\left|c_{347}\right|^{2}+\left|c_{125}\right|^{2}+\left|c_{457}\right|^{2}+\left|c_{126}\right|^{2}+\left|c_{467}\right|^{2} \leqslant(r-1) \mathcal{D}_{7}^{2}$. It is now clear that

$$
\begin{aligned}
\left\|P_{7} \Phi\right\|_{2}^{2} & =1-\left(L+\left|c_{347}\right|^{2}+\left|c_{125}\right|^{2}+\left|c_{457}\right|^{2}+\left|c_{126}\right|^{2}+\left|c_{467}\right|^{2}\right) \\
& \geqslant 1-(r-1) \mathcal{D}_{7}^{2}=1-\frac{1+9\left(1-|\epsilon|^{2}\right)}{1-11\left(1-|\epsilon|^{2}\right)} \mathcal{D}_{7}^{2} \geqslant 1-\frac{1+9 \xi}{1-11 \xi} \mathcal{D}_{7}^{2},
\end{aligned}
$$

where in the last inequality we have used $1-|\epsilon|^{2} \leqslant \xi=3-\lambda_{1}-\lambda_{2}-\lambda_{3}$, which is Lemma 3 in [14]. 
[1] R. E. Borland and K. Dennis, J. Phys. B 5, 7 (1972).

[2] M. B. Ruskai, J. Phys. A 40, F961 (2007).

[3] M. Altunbulak and A. Klyachko, Commun. Math. Phys. 282, 287 (2008).

[4] D. A. Mazziotti, Phys. Rev. Lett. 108, 263002 (2012).

[5] C. Schilling, D. Gross, and M. Christandl, Phys. Rev. Lett. 110, 040404 (2013).

[6] P. Lévay and P. Vrana, Phys. Rev. A 78, 022329 (2008).

[7] M. Springborg, Methods of Electronic-Structure Calculations (Wiley, Chichester, UK, 2000).

[8] H. Shull and P.-O. Löwdin, J. Chem. Phys. 30, 617 (1959).

[9] N. N. Lebedev, Special Functions and Their Applications (Dover, New York, 1972).
[10] A. J. Coleman, Rev. Mod. Phys. 35, 668 (1963).

[11] L. Chen, J. Chen, D. Ž. Djoković, and B. Zeng, arXiv:1301.3421.

[12] L. Amico, R. Fazio, A. Osterloh, and V. Vedral, Rev. Mod. Phys. 80, 517 (2008).

[13] A. P. Balachandran, T. R. Govindarajan, A. R. de Queiroz, and A. F. Reyes-Lega, Phys. Rev. Lett. 110, 080503 (2013).

[14] Supplemental Material to Ref. [5].

[15] E. R. Davidson, Reduced Density Matrices in Quantum Chemistry (Academic Press, New York, 1976).

[16] C. L. Benavides-Riveros and J. M. Gracia-Bondía, Phys. Rev. A 87, 022118 (2013). 